\title{
INFLUENCES OF A NEW DIGITAL CULTURAL LAYER ON DESIGN AT VARYING SCALES
}

\author{
JAMES HOLTH ${ }^{1}$, SCOTT MEEKINGS ${ }^{2}$, \\ MARC AUREL SCHNABEL ${ }^{3}$ and TANE JACOB MOLETA ${ }^{4}$ \\ ${ }^{1}$ PLUS Architecture \& DARA \\ 1james.r.holth@gmail.com \\ ${ }^{2} D A R A$ \\ ${ }^{2}$ s.m.meekings@gmail.com \\ ${ }^{3,4}$ Victoria University of Wellington \\ ${ }^{3,4}\{$ MarcAurel.Schnabel|Tane.Moleta\}@vuw.ac.nz
}

\begin{abstract}
Architects work with data daily. Spatial metrics, building codes and client requirements form the main considerations for many designers, yet new layers of data are impacting the way cities and inhabitants interact with each. This data can be used to more effectively analyse and predict patterns and behaviors to produce environments better suited to users. This paper reviews a selection of ideas from across digital architectural discourse by discussing tangible outcomes from a practitioner point of view and advocates for a greater integration of this digital cultural context into the design process. This paper considers a city-wide digital logic, rather than a new-age technological zeitgeist, that is as much a part of a city as its buildings are and through this provides a lens into our environment and devices that can be used to influence design at multiple scales.
\end{abstract}

Keywords. Big Data; Digital Identity; Built Environment; Authenticity.

\section{Big Data, Small World}

"The future of architecture is culture." - Philip Johnson

A cities culture is a product of the lifestyle of its people. It is an organic system that reacts to and in turn influences people. Architecture and the built environment form an intrinsic part of this system. Not only does the designed environment provide the physical amenity for many of a cities activities, they keep a record of a cities past. The built environment has an important role to play by being receptive to and enhancing a cities culture in order to help guide its future. This requires a proactive approach by architects that considers how architecture is situated in a cultural timeline by developing academic considerations into additional constraints and motivators for practice.Today's society collects data on an unprecedented level (Neef, 2014). Interaction with data has moved from a purely practical level, i.e. maps, bus schedules, to include more whimsical uses, i.e. location based mobile games. Data collection has become a normalised

Intelligent \& Informed, Proceedings of the $24^{\text {th }}$ International Conference of the Association for Computer-Aided Architectural Design Research in Asia (CAADRIA) 2019, Volume 2, 373-380. (C) 2019 and published by the Association for Computer-Aided Architectural Design Research in Asia (CAADRIA), Hong Kong. 
aspect of many peoples lifestyles (Mayer-Schonberger, 2013). Lifestyle apps have enabled large scale personal data collection, analysis and comparison; with many of these apps tied to location, see Figure 1 for an example of simple user location tracking. These data collection apps are becoming increasingly linked to social media, providing means to interact and connect with nearby users. In the same way that a park, or a popular bar, may have connected a cities people in the past, these apps are connecting people to different locations in a new way. As this becomes more prevalent, and reaches an international scale, traditional wayfinding techniques will blur with more data driven approaches that can be harnessed by viable developments.

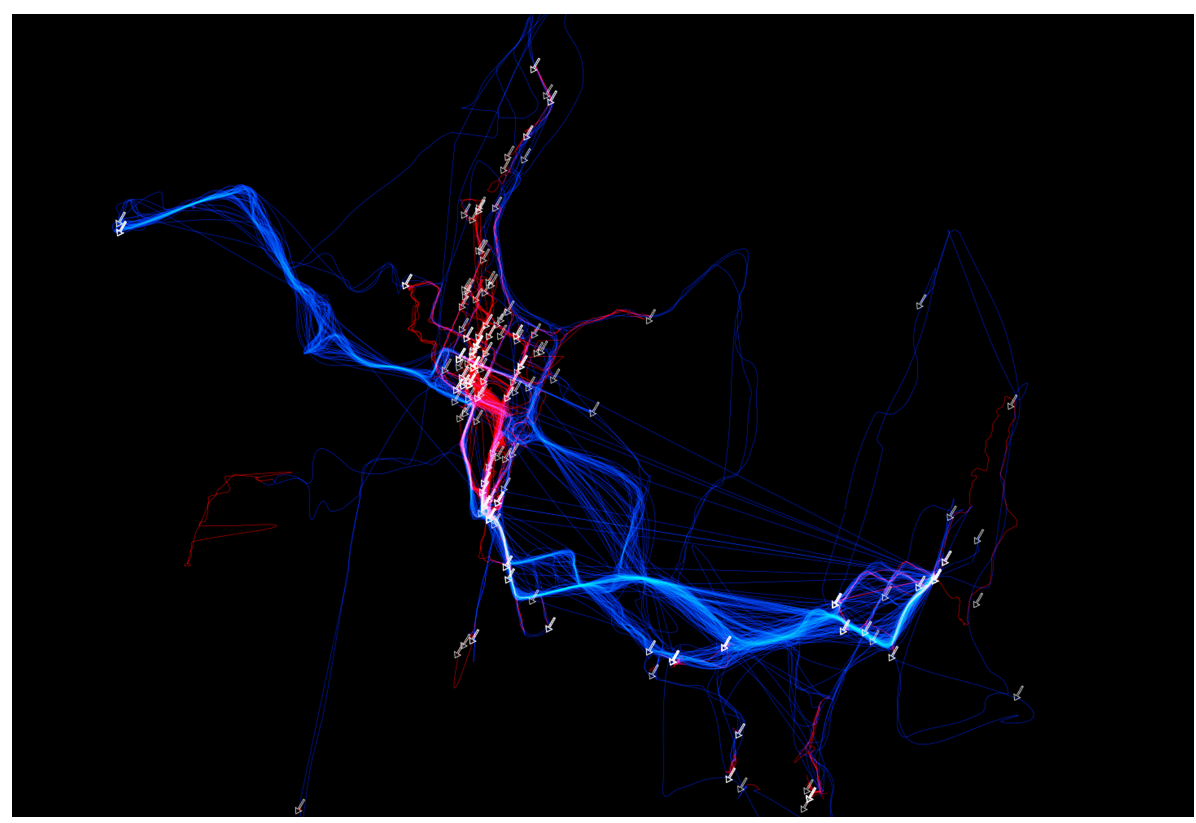

Figure 1. Exploration of personal datasets within cities Source: Author.

There is room for greater integration of the digital environment into the design process in order to create spaces that are better integrated into a digital cultural context. Each city has its own intelligence in the form of patterns, and it is important to view this data not simply as sets of disparate data, but as a layer of aggregated culture.

As the way we interact with the environment has shifted, so too have the environments themselves. Businesses are putting more information online. Tourist destinations are being artificially framed for social media, see Figure 2. 


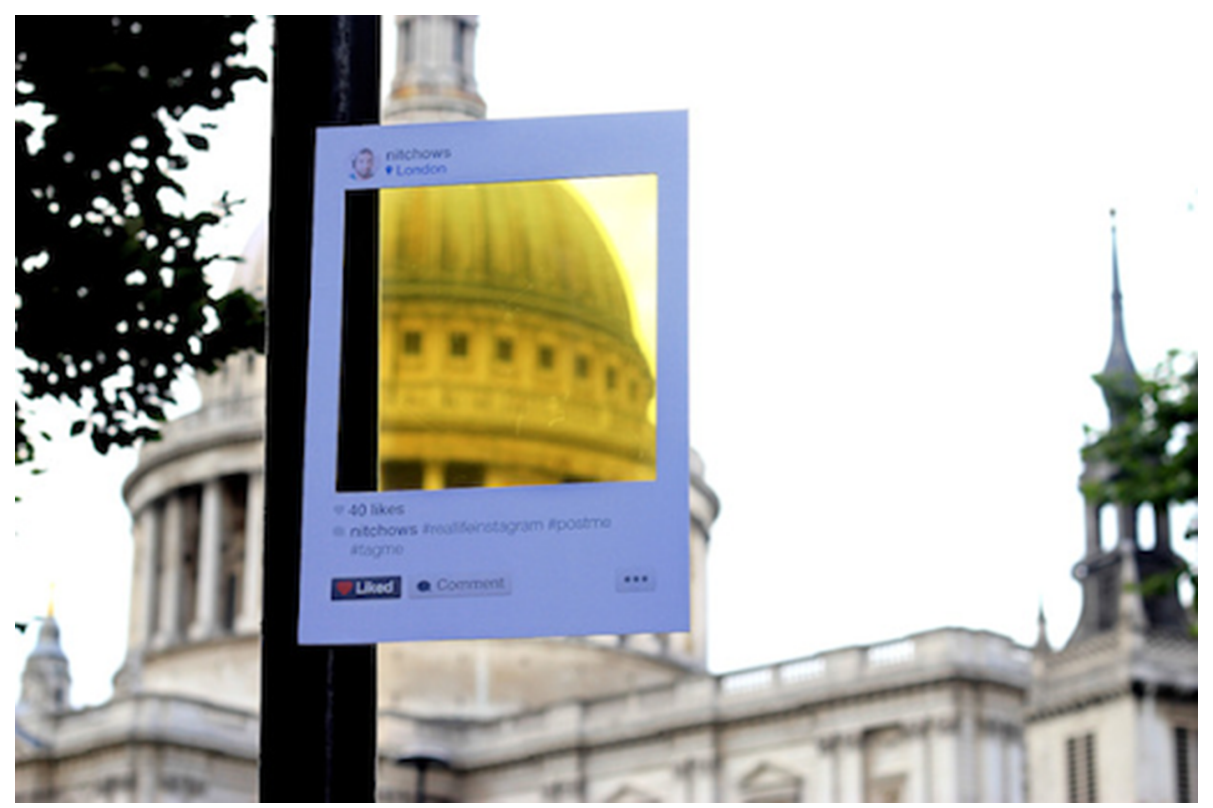

Figure 2. Real Life Instagram project Source: Bruno Ribeiro.

Cafe's are including USB ports to charge devices, and WiFi to browse social media. Even public spaces are beginning to incorporate aspects of our increasingly digital culture (Figure 3). Our environments are showing signs of adapting to this shifting cultural paradigm.

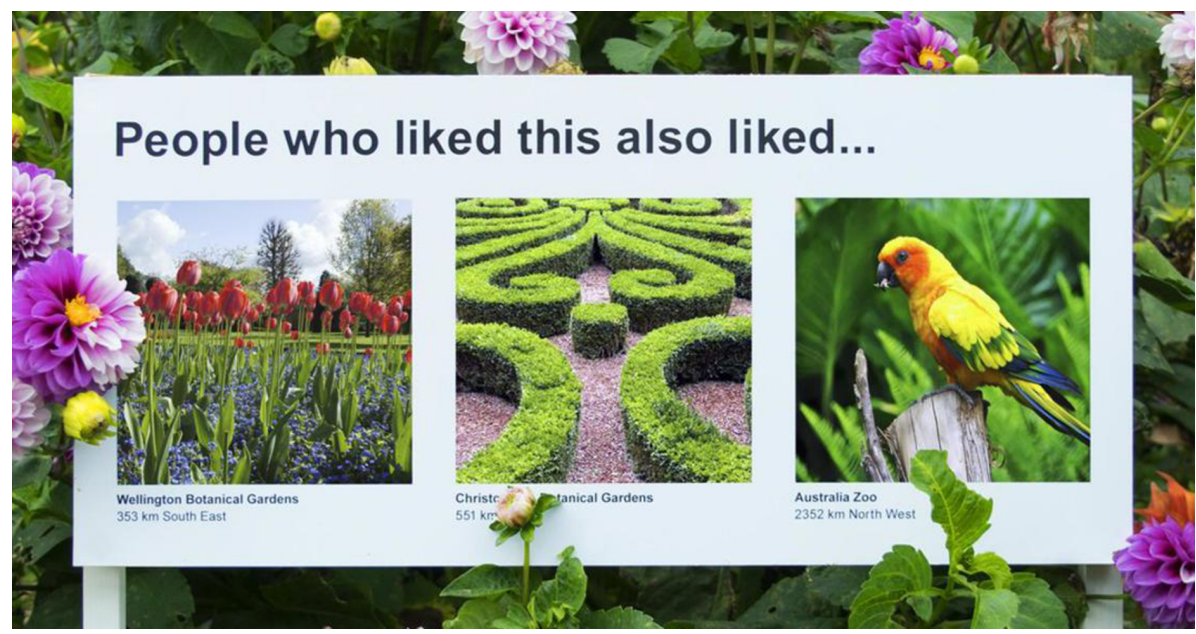

Figure 3. Signs of the Times project Source: Scott Kelly and Ben Polkinghorne.

In order to explore this concept, two scales have been considered; urban scale and human scale. The first scale questions the influence of this cultural layer when 
applied on a city scale, where people are represented as data points and trends. This section discusses the strengths and weaknesses of a holistic data-driven planning perspective. The second scale introduces a relationship between buildings and people through data centred interaction. The idea of a digital culture layer is examined at an impersonal as well as a personal level.

\section{Urban Scale}

Data has been collected at an urban scale for a long time; however, the level of available data is much greater than before. This allows for insights to be gained into different aspects of how people live in a city. Large amounts of data and layers of information are becoming integrated into city programs in a network of interoperability but that is still only on the fringe of practical usage. Shifts in the scale and interoperability of this digital network are constantly changing the applications for this data, and providing increasing possibility to influence design at a greater scale.

Site and context are key factors to consider for a successful architectural outcome, and the digital cultural layer provides additional tools and complexities for engaging with site. Aggregate data of digital interaction can quantify conventional values such as mood, age, sex and wealth. More importantly, aggregate data can attempt to provide additional considerations such as a communities shared personality traits (Chittaranjan et al., 2013). Similarly the ability to derive a sense of a communities aesthetic taste is increasingly possible as the digital cultural layer comiles individual preferences. Through aggregate data, site can be informed by digital data at an urban scale in much the same way as traditional site influences.

This is exhibited in projects such as the Hemnet Home by Tham \& Videgård Arkitekter where the 'ideal' house was designed based on the browsing and buying habits of users of Swedish property buying website, Hemnet (2015). This provided data on preferences such as prefered colours and configuration of rooms. Additional digital datasets can be integrated with these location specific information sources to provide a macro-scale context which can see wider societal changes such as year on year declines in searches for apartments. These patterns can be compared across cities giving context to any location, see Figure 4. This use of aggregated data can allow a city to make an informed response to growing trends using the digital space before the're manifested physically. The ability to integrate a quantitative view of trends or tastes allows for a more direct relationship to clients and developer goals. 


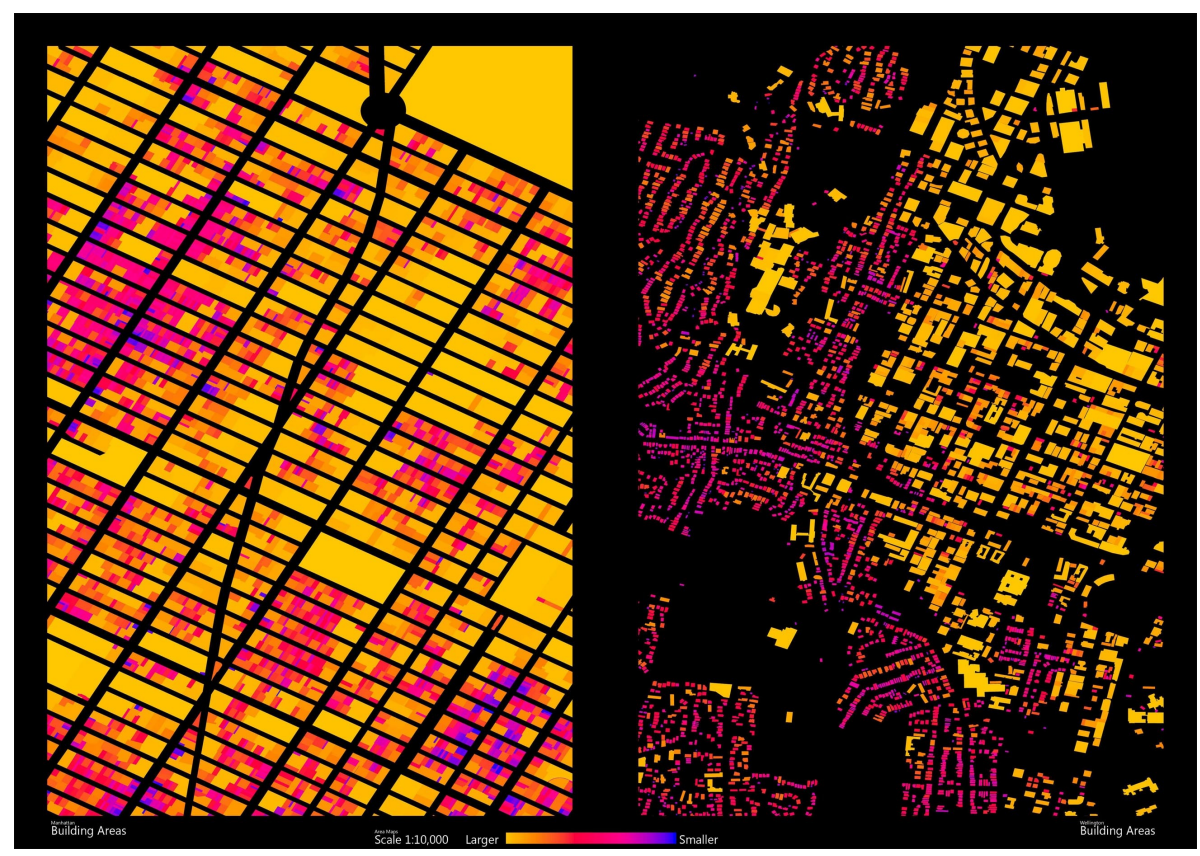

Figure 4. Exploration of patterns and datasets within cities Source: Author.

A data driven approach is not without its pitfalls. The dichotomy of a need for, and danger of, a centralized framework is one area that needs careful consideration. Privacy issues around the widespread use of personal data also create an ethical element to its use (Neef, 2014).

There are difficulties associated with aggregating data such as creating a dashboard or visualization to make sense of a cities complexity under the guise this will make for easier decision making - however this view advocates for a framework that is designed, operated and controlled without conscious input from those affected (Ciuccarelli, et al., 2014). By ignoring these stakeholders the outcome is likely to diminish the culture which it is based upon. This is the inherent risk in the filtering stage of data-driven design. In doing so, user bias can allow for pre-conceived results to be falsely justified. It is important that the use of data is a transparent process that allows organic solutions to form, where people act more as observers and less as operators.

Contrary to the issues of too much centralized control is the inherent reliance on existing centralized infrastructure frameworks. This reliance puts these intermediary government or private organisations (governmental or private) in the powerful position of influencing the built environment, due to their ability to curate and diseminate the aspects of the digital cultural layer known as "gatekeepers" (Segev, 2010). Alongside the need for centralized frameworks is the need for oversight of data, as unexpected issues can arise from the release and publicization of data which has not been vetted. An example of these issues was spurred by Strava publicizing popular running routes and unknowingly identifying US 
military base locations (Ruser, 2018).

A further issue with centralizing control is the need to anonymize data without removing key outliers. Design professions differ from typical data-driven professions in that we deal with the creation and reaction to unusual and atypical inputs as opposed to considering them irrelevant and removing them as outliers. This makes privacy more difficult to maintain as uniqueness is quintessential to the built environment but is also directly antithetical to maintaining privacy (Meekings and Schnabel, 2016).

\section{Personal Scale and Agency}

Connection and interactivity help make a space successful. Personal interaction with the digital cultural layer is changing as it becomes a part of the built environment. Typically users have agency over their digital interaction and profile, often referred to as an avatar. As data is being collected and curated to reflect individuals this agency is removed. Explored here is an opposition to this reduced agency through the ability for individuals to filter how data's influence is manifested in physical space.

When interacting with the digital environment, individuals have become accustomed to the concept of a digital avatar that represents them in a digital setting. The separation of data into an 'avatar' to represent an individual is common throughout digital technology; a set of preset details that, for convenience's sake, carry across multiple media. This concept can be seen in physical space through profiles used in home automation systems that enable occupants to adjust lighting per room, monitor their home from other locations and set presets that recognise an occupants personalized settings. This avatar typically gives agency to the user, as they have the ability to modify or control how their avatar represents them.

As the built environment adapts to incorporate digital culture, it too will start to connect with these digital identities. As lifestyles become so intrinsically linked with digital personas, the distinction between 'avatar' and individual will become blurred. Once digital interaction becomes a part of physical space, the need for an avatar disappears, as data can be directly associated with the individual.

Through leveraging existing methods of profiling, smart buildings and automation will be able to associate data with individuals without the need for user input. This is different to a user-created avatar in that the profile is created without agency from the user. These existing methods are currently used in moderating and curating the digital cultural layer by internet-based marketing and social media companies, which rely on their ability to extract data from digital interactions and use this to form a profile representing an individual. This profile can help digital organizations make assumptions on the preferences of an individual and deliver a personalized experience within a wider framework. Despite there being no agency in creating this profile, this data is still being made accessible to individuals; google for example provides a webpage for reviewing their assumptions about you while spotify will allow you to download a list of every song you have listened to via their service. By leveraging these existing data-sources and algorithms a plethora of architectural outcomes become available. Examples vary widely from simple 
INFLUENCES OF A NEW DIGITAL CULTURAL LAYER ON DESIGN 379 AT VARYING SCALES

ideas such as intuitively dimming and reducing the blue-wavelength of lights based on an individual's typical sleeping patterns ("blanched" melatonin levels are an increasing problem due to the digital cultural layer and it's current reliance on constant connectivity and backlit screens) (Scott, 2016). More complex options are made available with more data, examples include real-estate or apartments being coordinated to create symbiotic communities of people based on these profiles, or recommending areas to live based on facilities and locations most visited as recorded by phone GPS (Meekings, 2017).

As an individual's agency is removed through the replacement of 'avatars' with automatically generated profiles, this agency could be restored by giving the user increased input into how data influences the built environment around them; likely in the form of reducing the data that is available to them in physical space rather than the inclusion of more data. In the past this area of research has largely focussed on overlaying and increasing the amount of data that is made available through architectural space, with early examples such as MIT tangible media groups Ambient Room which utilised interactions that were novel for the time such as projections and physical icons referred to as phycons (Wisneski et al., 1998). This has been a continual theme in this specialism such as the more recent BMW Museum by ART $+\mathrm{COM}$ (Dalton, et al. 2016). Increasingly there is a sense that 'data overload', limitless access and constant connectivity can have negative emotional or physical outcomes. It is increasingly impossible to be 'fully-informed' as the internet-enabled pool of data and information becomes vastly deeper than ever before. If data and information become synonymous with the built-environment around us this sense of 'data-overload' will reach new heights. This 'data' overload' can have profound effects on wider society, politics and productivity; factors that are often associated with the built environment but often only sparingly considered by designers (Andrejevic, 2013).

It is important to understand a personal digital context to both facilitate and provide relief from data driven interactions. This could be a way to give agency back to users as it does not focus on reducing the data that is associated with a profile. Environments designed to create a relaxed atmosphere could act as filters for some of this information. Existing examples of a data-restricted environments are no-reception zones within offices and libraries to ensure certain areas are free from phone calls and an increasing awareness of designing for quiet work (Cain, 2012). Through a moderated level of agency, spatial occupants can consciously review and edit how they interact with their environments and provide an experience that both protects and enhances the way they experience their surroundings.

\section{Conclusion}

Successful architecture as a discipline and built work should interface with the digital cultural layer at multiple scales. The evolution of a digital cultural layer provides a basis for changes to the architectural discipline, structured around academic ideas of urban and human interaction. A cities culture is tied to its built environment, therefore the built environment should be responding to shifts in digital as well as physical culture. 
This paper assesses the way digital culture has and can influence the built environment, and in what way advances in digital architectural knowledge can influence practice. At an urban scale, this paper puts forward ideas centered on the role of centralized frameworks and associated pitfalls. Issues discussed at a personal scale center on changing dynamics of agency and the role of 'avatars'.

Overall this paper seeks to promote the further exploration of the myriad of ways our increasingly evolving digital culture has impacted our cities, and our buildings. It advocates for a considered approach to both urban planning and building design as the digital cultural layer makes its way into mainstream practice. Interaction with a cities culture is a key to the designed environments success at any scale. Our culture is shifting; as are our cities, in order to continue designing successful spaces we must too.

\section{References}

Andrejevic, M.: 2013, Infoglut, Routledge. 2013..

Tham \& Videgård Arkitekter, N./A.: 2015, "The Hemnet Home" . Available from <http://ww w.tvark.se/the-hemnet-home/>.

Cain, S.: 2012, Quiet: The power of introverts in a world that can't stop talking, New York: Crown.

Chittaranjan, G., Blom, J. and Gatica-Perez, D.: 2013, Mining large-scale smartphone data for personality studies, Personal and Ubiquitous Computing, 17(3), 433-450.

Ciuccarelli, P., Lupi, G. and Simeone, L.: 2014, Depicting the Data City, Springer.

Dalton, N.S., Schnädelbach, H., Wiberg, M., Varoudis, T., Dalton, N.S., Schnädelbach, H., Wiberg, M. and Varoudis, T.: 2016, Architecture and interaction : human computer interaction in space and place, Switzerland : Springer. 2016.

Kelly, S. and Polkinghorne, B.: 2017, Sign of the Times, [Artwork].

Mayer-Schönberger, V. and Cukier, K.: 2013, Big Data-A Revolution That Will Transform How We Live, Think and Work, $N / A$.

Meekings, S. and Schnabel, M.A.: 2016, Big Data on Individuals in the Architectural Design Process: Combining Individual's Data with the Architects Toolset, International Journal of Parallel Emergent and Distributed Systems, 32, 66-72.

Meekings, S. and Schnabel, M.A.: 2017, Datatecture: Creating a real home for a virtual identity, Master's Thesis, Victoria University of Wellington.

Neef, D.: 2014, Digital Exhaust: What Everyone Should Know About Big Data, Digitization and Digitally Driven Innovation, Pearson Education.

Ribeiro, B.: 2013, Real Life Instagram, [Artwork].

Ruser, N. [@N.R.G.8000]: 2018, "Strava released their global heatmap. 13 trillion GPS points from their users (turning off data sharing is an option). It looks very pretty, but not amazing for Op-Sec. US Bases are clearly identifiable and mappable [Tweet]". Available from $<\mathrm{ht}$ tps://twitter.com/nrg8000/status/957318498102865920?lang=en>.

Scott, L.: 2016, The four-dimensional human: Ways of being in the digital world, Random House.

Segev, E.: 2010, Google and the digital divide: The bias of online knowledge, Elsevier.

Wisneski, C., Ishii, H., Dahley, A., Gorbet, M., Brave, S., Ullmer, B. and Yarin, P. 1998, Ambient displays: Turning architectural space into an interface between people and digital information, in N. Streitz, S. Konomi and H.J. Burkhardt (eds.), Cooperative buildings: Integrating information, organization, and architecture, Springer, 22-32. 